\title{
A Study of the Stress Induced during Manufacturing Processes of Chip Inductors
}

\author{
Yin-Lai-Chai ${ }^{1}$, Shih-Feng Chien ${ }^{2 *}$, Wen-Ting Chen ${ }^{2}$, Wen-Hsi Lee ${ }^{2}$, Pei-Yi Wei ${ }^{3}$, Wen-Yu Lin ${ }^{3}$ and Po-Chih \\ Shen $^{3}$ \\ 1. Department of Jewelry Technology, Dahan Institute of Technology, Hualien County 97145, Taiwan (R.O.C.) \\ 2. Department of Electrical Engineering, National Cheng Kung University, Tainan 701, Taiwan (R.O.C.) \\ 3. Cyntec Co., LTD., Hsinchu County 30076, Taiwan (R.O.C.)
}

\begin{abstract}
Ni-Cu-Zn ferrite components are very sensitive to stress and this can cause unstable permeability. This work focuses on the source of stress, and the interaction between the residual stress after sintering and the further stresses contributed by subsequent manufacturing processes of multilayer chip inductors. The results show that the sources of stress include cofiring of ferrite and silver coils, and the operations involved in the manufacturing processes. The results also show that the stresses pass through the interfaces between materials to the body of component and that the stresses induced in the subsequent manufacturing processes would accumulate or counteract with the residual stress in the component, although the stress can also be released by changing the connection status of these interfaces. The results also show that the compressive and tensile stresses cause different levels of inductance shift.
\end{abstract}

Key words: Magnetic, multilayer, stress.

\section{Introduction}

Due to the high anisotropy constant and magnetostriction constant of magnetic materials, their permeability has a close relation with stress. The magnetic field contributes to the permeability until it overcomes the anisotropy and magnetostriction characteristics, and thus a number of articles treat these characteristics as energy losses, and note that the anisotropy constant [1] and magnetostriction constant [2-5] can be reduced by doping with minor elements. One other study examines the relation between microstructure, stress and permeability, and demonstrates that changing the grain size can minimize the stress and obtain higher inductance [6]. To achieve the need for further miniaturization, manufacturers have started to apply a multilayer process to magnetic components, and one of the key

Corresponding author: Shih-Feng Chien, Ph.D. candidate, research fields: magnetic materials and multlayer process. E-mail: eddie.chien@cyntec.com. processes in producing multilayer chip inductors is cofiring of the coil and core, in which the materials are silver and $\mathrm{Ni}-\mathrm{Cu}-\mathrm{Zn}$ ferrite, respectively, and because of their shrinkage differences, a significant and complicated stress system is built up in this process [7, 8]. Some articles note that the $\mathrm{Cu}$ element of $\mathrm{Ni}-\mathrm{Cu}-\mathrm{Zn}$ ferrite reacts with the $\mathrm{Ag}$ of coil, segregates in the grain boundary and forms a local stress $[9,10]$ during cofiring. In addition, the $\mathrm{S}$ and $\mathrm{Cl}$ impurities in the raw material would react with $\mathrm{Ag}$ to form a low melting compound and make the $\mathrm{Ag}$ element diffuse in sintering, which increases the possibility of reactions occurring with $\mathrm{Cu}[11,12]$.

In addition to sintering, during the manufacturing processes for chip inductors, the silver, nickel and tin layers are used as the terminations on the surface of components, and their shrinking during the related processes affects the stress inside the chip inductor [13-15], similar to what is seen during sintering, and this causes a shift in inductance. 
Understanding the mechanism and nature of stresses would help us to control them in order to stabilize the inductance of chip inductors, and although there are many studies of stress characteristics of magnetic materials, there are few that examine the construction and manufacturing process of chip inductors. This work thus focused on $\mathrm{Ni}-\mathrm{Cu}-\mathrm{Zn}$ ferrite, cofiring, and the construction and manufacturing processes of chip inductors to uncover the cause, distribution and release of stresses, in order to develop a method to avoid or eliminate these.

\section{Experimental Setup}

\subsection{Sample Preparation}

$\mathrm{Ni}-\mathrm{Cu}-\mathrm{Zn}$ ferrite powder (with the composition shown in Table 1) was used to prepare the slip and foil, and then the silver paste (Table 2) was printed on foils for 8.5 turns as coils, and then stacked, pressed, and cut into pieces with the dimensions of $2.5(1) \times 2.0(\mathrm{w})$ $\times 1.0(\mathrm{t}) \mathrm{mm}$. These samples were fired at $870{ }^{\circ} \mathrm{C}$ and $895{ }^{\circ} \mathrm{C}$ for $1 \mathrm{~h}$, and then dipped and cured at $700{ }^{\circ} \mathrm{C}$ for $40 \mathrm{~min}$ and plated to form the terminations.

\subsection{Measurements}

The samples prepared in step 2.1 were then used for the pull and shear tests. The copper wires were jointed at each end of the component to connect to the LCR (inductance-capacitance-resistance) meter. Before starting the shear test, both ends of component were soldered on two different boards and the instrument pushed the boards, and then the results were obtained as the inductance under different levels of compressive stress. Before starting the pull test, one end of the component was soldered on a board to be fixed during the test, and the other end was jointed to a wire to be pulled by the instrument, and the results were obtained as the inductance under different levels of tensile stress.

The inductance of the samples from step 2.1 was measured after every process and after the TC (thermal cycling) treatments $\left(-55 \sim 125^{\circ} \mathrm{C}\right.$, with a soaking
Table 1 The composition of $\mathrm{Ni}-\mathrm{Cu}-\mathrm{Zn}$ ferrite.

\begin{tabular}{lllll}
\hline Composition & $\mathrm{Fe}_{2} \mathrm{O}_{3}$ & $\mathrm{NiO}$ & $\mathrm{CuO}$ & $\mathrm{ZnO}$ \\
\hline $\mathrm{Mol} \%$ & 48.3 & 15.3 & 9.7 & 26.7 \\
\hline Table 2 & The data of the silver pastes. \\
\hline Code & Solid Content $(\%)$ & \multicolumn{2}{l}{ Particle size $(\mu \mathrm{m})$} \\
\hline Paste & 87.5 & 2.5 & \\
\hline
\end{tabular}

time of $30 \mathrm{~min}$ for each peak temperature, and 10 cycles for a single treatment), then the change of inductance was calculated.

\subsection{Observations}

The samples from step 1 were soaked in tin plating solution $(\mathrm{pH}=4.5)$ for 12 and $24 \mathrm{~h}$.

\section{Experiment Results}

Shear and pull forces were applied to the chip samples to simulate the stress, and the inductance level was measured under different levels of stress, with the results shown in Fig. 1. It is clear that the inductance trend is very different and asymmetrical for the compressive and tensile sides. On one hand, there is a small area of tensile stress that had a $1 \%$ increase in inductance as reported in an earlier study [11], and the other stresses, both compression and tension caused a fall in inductance. On the other hand, the rate of change of compressive stress is smaller than that of tensile stress, and thus if the stress is unavoidable, then it might be controlled in the compressive stress region to obtain a stable inductance level.

The results presented above show the relation between inductance and stress, and that the source of stress and potential location of stress release are the interfaces between materials inside the components. Furthermore, they reveal that the manufacturing processes following sintering would also alter the inductance.

The inductance trend of the manufacturing processes is shown in Fig. 2 for the components which fired at $870{ }^{\circ} \mathrm{C}$. The inductance first went down after curing process and then rose significantly after Ni-plating, and then slightly after Sn-plating. With 


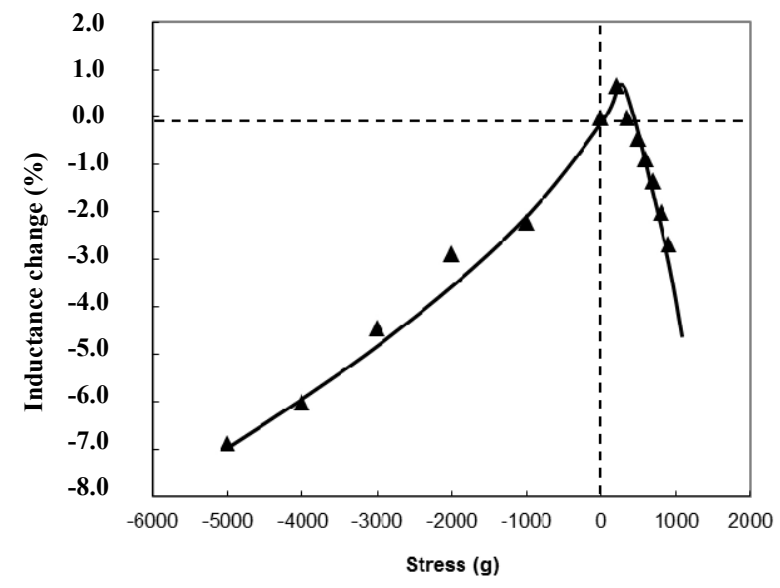

Fig. 1 The characteristic plot of inductance-stress.

regard to the inductance change after TC treatment for each process, the curing saw a $13 \%$ rise, while Ni-plating and Sn-plating saw 5\% and $8 \%$ increases, respectively. The change in inductance after each process could be considered as involving new stresses. There are two possible sources of these new stresses, the first is mechanical stress or expanding/shrinking behavior from heating during the process, and the effect of this type of stress is temporary. The second source is from the added materials attaching to the surface of the components and applying extra stress to the body of the components, and this type of stress would react with the original stress and remain permanently as residual stress inside the components.

As mentioned above, the silver coil and ferrite core of chip inductors were cofired during sintering. Because these two materials have a significant mismatch of shrinkage rate, and the Ag element of the coil reacts with the $\mathrm{Cu}$ element of the core and then segregates at grain boundaries, and this will build up the comprehensive and local stresses, which remain as residual stress and reduce the inductance level. Therefore, when these components underwent TC treatment, some of the residual stress could be released and the inductance would be increased. In addition, when we measured the components after the curing process, the inductance level dropped and then the inductance increased after TC treatment, compared with the levels after sintering, and this reveals that both

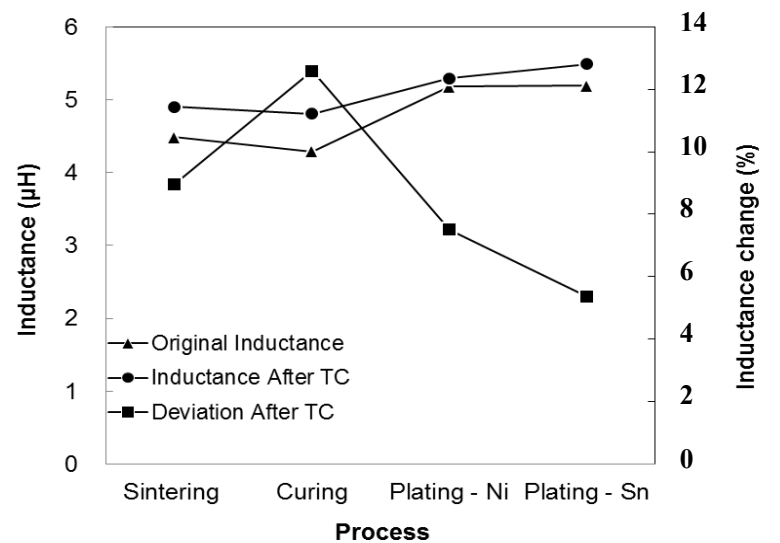

Fig. 2 The inductance levels of each process when the components were fired at $870{ }^{\circ} \mathrm{C}$.

sintering and curing processes increased the residual stress level to reduce the inductance level, and also means that the stress produced by curing was the same type as that produced by sintering, and that it accumulated inside the components. In contrast, compared to the fall in inductance after the curing process, the inductance increased significantly after $\mathrm{Ni}$ - and Sn-plating. The Ni and Sn layers which were produced during these plating processes built up the stress $[14,15]$, and this would react with the residual stress inside the components.

Nevertheless, if the stress level is increased inside the components, the inductance level must be lowered, not increased. We have three hypotheses to explain this inconsistency. The first one is that the stress from the plating process was counteracted by the residual stress inside the components, as to directions of these two stresses were opposite, resulting in an increase in inductance after the plating process. The second is the corrosive effect of plating solution, which causes the inductance to increase. The third is that a current was applied during plating, and this could create a magnetic field in the ferrite core of the components and change the inductance level after plating. Furthermore, the Ni-layer produced during plating process is also a magnetic material, and this might react with the magnetic field of the ferrite core, leading to the different results found for $\mathrm{Ni}$ - and Sn-plating. The factors that cause the change in the 
inductance level are more numerous and complicated than those involved in other processes, and more study is needed to address the contribution of each factor. However, we now know that the change in inductance after plating is a net effect of these three factors.

The samples which were fired at $895^{\circ} \mathrm{C}$ were then used for the same measurements and observations as those fired at $870{ }^{\circ} \mathrm{C}$, and the results are shown in Fig. 3. It can be seen that whole inductance trend of the $895^{\circ} \mathrm{C}$ fired component is completely different from that of the $870{ }^{\circ} \mathrm{C}$ one, and the inductance level of former is greater than that of latter. In the components made of pure ferrite, a higher sintering temperature reduces the porosity and enlarges the grain size, and both of these phenomena reduce the difficulty of magnetic moment rotation and magnetic domain wall motion, resulting in increased permeability. After TC treatment of the $895{ }^{\circ} \mathrm{C}$ fired component, the inductance change was $15 \%$, and higher than the $9 \%$ found with the $870{ }^{\circ} \mathrm{C}$ fired component.

The TMA (thermomechanical analysis) data for the silver coil and ferrite core are shown in Fig. 4, and the difference in their shrinkage rates could build up the stress during sintering. Comparing with the status at $870{ }^{\circ} \mathrm{C}$ and $895{ }^{\circ} \mathrm{C}$, the mismatch difference and magnitude of silver and ferrite changed significantly; that is, when the sintering temperature was higher, not only did the shrinkage difference change, but also their magnitude, with that of ferrite becoming greater than that of silver. Therefore, we can deduce that the part of the permeability that came from microstructure might be counteracted by the extra stress coming from greater mismatch of shrinkage when the sintering temperature is raised, and the TC treatment released much more stress and increased the inductance. For the following processes, the inductance was increased after the curing process and was reduced after plating, and this was due to a number of causes. However, one certainty is that even when the sintering temperature changed, it did not affect the manufacturing conditions of the following processes at all, and this means that all

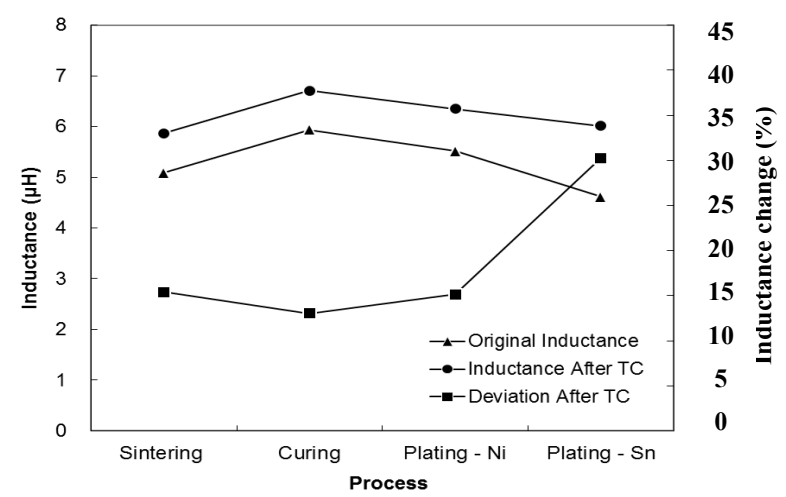

Fig. 3 The inductance levels of each process when the components were fired at $895{ }^{\circ} \mathrm{C}$.

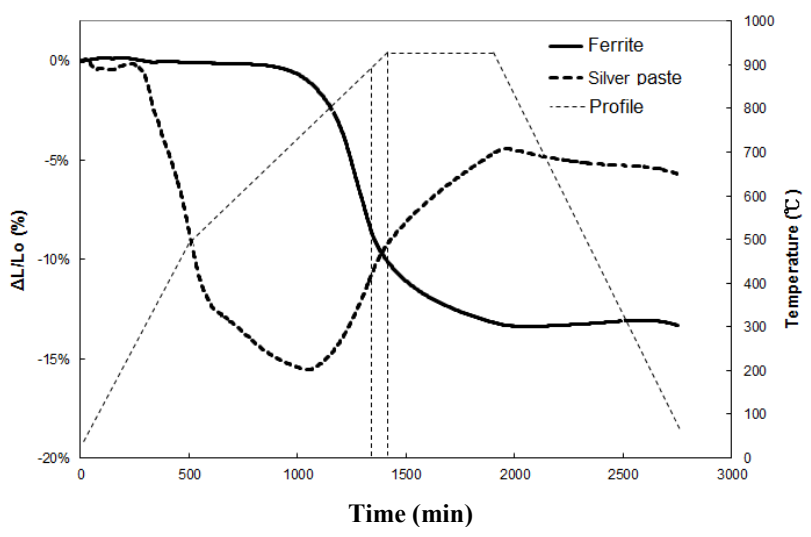

Fig. 4 The TMA of ferrite foil and silver paste.

of the effects from the temperature, mechanical stress and size of the extra materials on the components in these processes, and the stresses exerted on the components must remain the same.

The TMA data in Fig. 4 show that the changes in the relative conditions between silver and ferrite might change the type of residual stress inside the components. Therefore, we can deduce that the residual stress of the $870{ }^{\circ} \mathrm{C}$ fired component was compressive whereas that of the $895{ }^{\circ} \mathrm{C}$ fired component was tensile stress. This might be the causes of different inductance level trends for these two components. We can further deduce the stress trend by combining the inductance trend of the manufacturing process and the characteristic plot of inductance and stress. According to the TMA results, when the sintering temperature was $870{ }^{\circ} \mathrm{C}$, the shrinkage rate of the silver coil was greater than that of the ferrite core. As a result, the ferrite core was 
under compressive stress after the sintering process. The inductance of the components after the process then fell, which means that the compressive stress increased continuously.

When the Ni- and Sn-plating were completed, the Ni- and Sn-layers were added to the ends of the components, and the inductance should fall because the stresses increased continuously. However, as noted earlier, not only plating layers, but also the current and solution affected the inductance level, therefore, the contribution of the current and solution might be greater than that of stress, and the net effect is to increase the inductance. On the other hand, when the sintering temperature was increased to $895^{\circ} \mathrm{C}$, as noted about the conditions of the processes following sintering, all the effects from these processes would be the same, and thus the trend of the inductance level for the $895{ }^{\circ} \mathrm{C}$ fired component after every process was opposite to that was seen for the $870{ }^{\circ} \mathrm{C}$ fired component, which means that they had opposite types of residual stress. That is, the residual stress of the 895 ${ }^{\circ} \mathrm{C}$ fired component was tensile stress, while that for the $870{ }^{\circ} \mathrm{C}$ fired component was compressive stress, and these would counteract, resulting in an increase in inductance after the curing process. However, the net effect of the plating process might be opposite to the stress effect of the curing process, and so the inductance fell again after the plating process.

The analysis presented above shows that the residual stress of the sintering process was greater than the total from the of curing and plating processes, and no matter how the stresses moved, they still moved within the region of their original type, that is, the stress of the $870^{\circ} \mathrm{C}$ fired component moved in the compressive stress region only, while that of the 895 ${ }^{\circ} \mathrm{C}$ fired component only moved in the tension stress region. In addition, although the level of stress induced by curing and plating should be constant, the levels of change in inductance for the $870{ }^{\circ} \mathrm{C}$ and $895^{\circ} \mathrm{C}$ fired components were very different, with the former much smaller than that of the latter.
Combining the motion of stress in only single region in this case, we can suggest that the ratio of inductance and stress in the compressive region was smaller than that in the tensile region, and this matches our characteristic plot of inductance and stress shown in Fig. 1. We can further say that our sample components are more sensitive to tensile than compressive stress. The reason why stress changes the inductance is magnetostriction [2-6], which is a characteristic of changes in size when applying a magnetic field to a bulk material, and the change in size relates to the magnetic moment rotation and magnetic domain wall movement following the direction of magnetic field. The stresses that are either inside or outside the bulk make the magnetic moment rotation and magnetic domain wall movement more difficult, and cause changes in the permeability or inductance. However, tensile stress tends to reduce the magnetostriction, whereas compressive stress tends to increase it, and thus the inductance characteristics of compressive and tensile stresses are asymmetrical, and the inductance is more sensitive to tensile stress than to compressive stress. This also reveals that if the stress cannot be avoided, then the final residual stress should be controlled in the compressive region, as components with a low sensitivity to stress and temperature can then be obtained.

Based on the results presented above, we can combine the inductance trend in the characteristic plots of inductance and stress to see the trend of change in the residual stress of the components. In Fig. 5 , (1) (4) represent the processes of sintering, curing, Ni-plating and Sn-plating, respectively, and the arrows located on the left side of the plot are the stress trend of $870{ }^{\circ} \mathrm{C}$ fired component during these processes, whereas those on the right side are the stress trend of the $895^{\circ} \mathrm{C}$ fired one. We can thus see that the stress from sintering is the largest of all the processes, dominating the trends of stresses and inductance. More importantly, (3) is the net effect of Ni-plating, and there is a significant difference between the results for 
the $870{ }^{\circ} \mathrm{C}$ and $895^{\circ} \mathrm{C}$ fired components, and this does

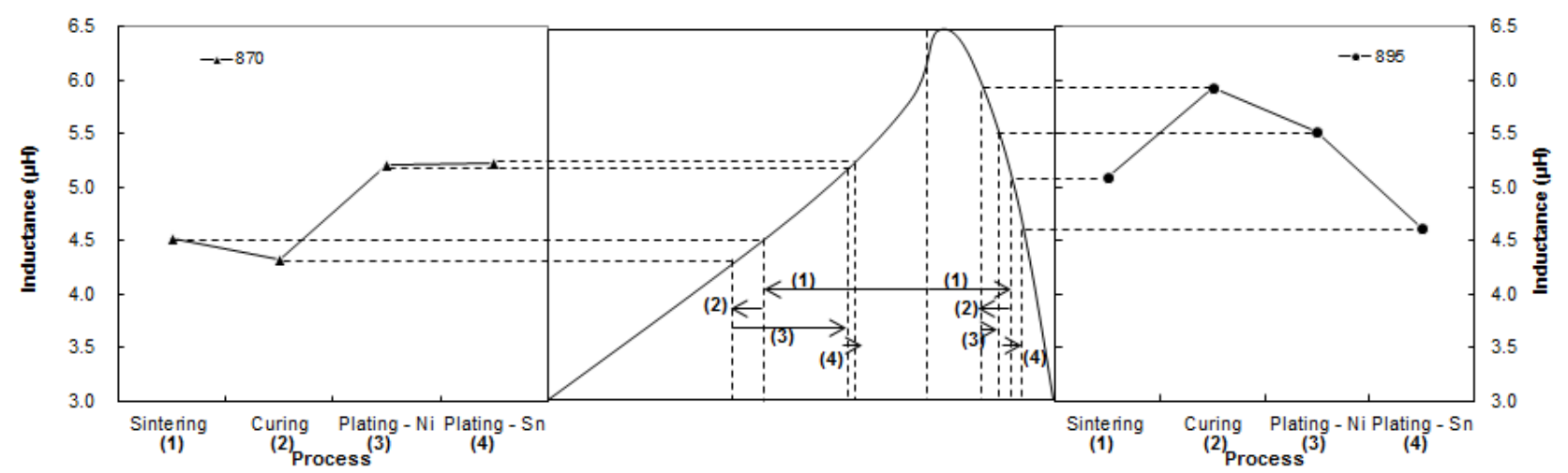

Fig. 5 A schematic diagram showing the inductance trend of components fired are $895{ }^{\circ} \mathrm{C}$ and $870{ }^{\circ} \mathrm{C}$ during sintering to Sn plating.

not correspond with our previous suggestion that the stress of the processes that occur after sintering are not affected by the sintering temperature. The reason for this inconsistency is because the effects of plating are so complicated, as not only the stress caused by the plating layers but also the magnetic effect caused by current and the corrosion effect caused by solution can all affect the inductance, and these effects should be studied further to address the contribution of each one of these.

\section{Conclusions}

The source of stress of the processes includes the sintering, curing and plating, and stresses delivery through the interface between the materials. However, we can change the status of the interface to cut off the connection between the materials and release the stress, and this might be the mechanism that occurs during TC treatment.

In addition, when the sintering temperature increased, the relative mismatch between the silver coil and ferrite core was changed, as was the type of residual stress. Because the stresses of the subsequent processes could not be changed by sintering temperature, so the accumulation or counteraction between new and original stresses inside the components would be changed if the relative shrinkage rate between silver coil and ferrite core was altered. The results also showed that the inductance of components was more sensitive to tension stress than compressive stress, and both characteristic plots of the inductance-stress and inductance change rate of the sintering to plating processes support this finding. Finally, with regard to the inductance sensitivity, if the residual stress can not be eliminated completely, then controlling the residual stress in the compressive stress region is more beneficial in this case.

Furthermore, the effects of plating are not only the plating layers causing stress, but also the current causing a magnetic field and the solution causing corrosion, and these all affect the inductance level after plating. Therefore, the plating process should be studied further to address the contribution of each of these factors.

\section{References}

[1] Reddy, N. R., Ramana, M. V., Rajitha, G., Rajagopal, E., Sivakumar, K. V., and Murthy, V. R. K. 2005. "Stress Sensitivity of Inductance in NiCuZn Ferrites." Journal of magnetism and magnetic materials 292: 159.

[2] Varalaxmi, N., Reddy, N. R., Ramana, M. V., Rajagopal, E., Murthy, V. R., and Sivakumar, K. V. 2008. "Stress Sensitivity of Inductance in NiMgCuZn Ferrites and Development of a Stress Insensitive Ferrite Composition for Micro Inductors.” J. Mater. Sci.: Mater Electron 19: 399.

[3] Reddy, N. R., Ramana, M. V., Gajitha, G., Sivakurmar, K. V., and Murthy, V. R. K. 2009. "Stress Intensive NiCuZn Ferrite Compositions for Micro Inductance Applications." Current Applied Physics 9: 317.

[4] Dionne, G. F., and West, R. G. 1986. "Nickel-zinc Microwave Ferrite with Stress-Intensitivity Square 
Hysteresis Loop.” Appl. Phys. Lett. 48 (21): 1488.

[5] Qi, X., Yue, J. Z., Gui, Z., and Li, L. 2002. "Effect of Mn Substitution on the Magnetic Properties of $\mathrm{MgCuZn}$ Ferrites." Journal of Magnetism and Magnetic Materials 251: 316-322.

[6] Drofenik, M., and Besenicar, S. 1986. "Reexamination of the Grain Size/Permeability Relation in High Permeability Mn-Zn Ferrites." Am. Ceram. Soc. Bull. 65 (4): 656-9.

[7] Kawano, K., Kusumi, S., Kishi, H., Fujii, A., and Mori, Y. 2007. "Lattice Distortion of NiZnCu Ferrite Co-Fired with Silver." Journal of Magnetism and magnetic materials 310: 2552.

[8] Ohiai, T. 1997. "Current Status of Soft Ferrite in Japan." J. Phys. IV France 7: 1-27.

[9] Nakano, A., Suzuki, T., and Momoi, H. 2002. "Development of Low Temperature NiCuZn Ferrites and Study of High Performance for Mulyilayer Chip Ferrites (in Japanese)." Journal of the Japan Society of powder and powder metallurgy 49(2): 77.

[10] Fujimoto, M. 1994. "Inner Stress Induced by $\mathrm{Cu}$ Metal
Precipitation at Grain Boundaries in Low Temperature-Fired Ni-Zn-Cu Ferrite.” J. Am. Ceram. Soc. 77 (11): 2873-8.

[11] Nakano A., and Nomura, T. 1999. "Multilayer Chip Inductors." Ceramic Transactions A. Cer. S. 97: 285-304.

[12] Nakamura, T., Okano, Y., and Miura, S. 1998. "Interfacial Diffusion between Ni-Cu-Zn Ferrite and Ag during Sintering." Journal of Materials Science 33: 1091-4.

[13] Fujikawa, N., Inagaki M., and Yokoe, N. 1990. "Simulation and Evaluation of Stresses in Surface Mounted Chip Capacitors." American Ceramic Society 8: 292.

[14] Keimasi, M., Azarian, M. H., and Pecht, M. 2007. "Isothermal Aging Effects on Flex Cracking of Multilayer Ceramic Capacitors with Standard and Flexible Terminations." Microelectronics reliability 47: 2215.

[15] Van Den Avyle, J. A. and Mecholsky, J. J. 1983. "Analysis of Soldering-Induced Crack of $\mathrm{BaTiO} 3$ Ceramic Capacitors.” Ferroelectrics 50: 293. 